\title{
RISK REDUCING MASTECTOMY IN PATIENTS WITH BRCA MUTATION: INITIAL EXPERIENCE
}

Sabas Carlos Vieira', Danilo Rafael da Silva Fontinele², Ana Lúcia Nascimento Araújo

${ }^{1}$ Oncocenter; Oncobem - Teresina (PI), Brazil.

Universidade Estadual do Piauí - Teresina (PI), Brazil.

Introduction: There are no randomized studies that assess the role of risk-reducing mastectomy in decreasing the mortality of mutated patients; however, major prospective studies show a decrease in the incidence of breast cancer. The absolute reduction in the risk of breast cancer ranges from $2 \%$ to $7 \%$, but the reduction of the risk of developing breast cancer is higher than $90 \%$. The increasing mortality caused by contralateral breast cancer in the mutated patient is $1.7 \%$, in 10 years, and 6.8\%, in 20 years. Objectives: To analyze the cases of risk-reducing mastectomy in patients with BRCA mutation. Method: This is a retrospective study. We included all patients who had positive BRCA mutation test, assisted from 1999 to 2019. The study was approved by the Research Ethics Committee of Universidade Federal do Piauí, report n. 2.817.502.

Results: We studied 23 patients who had a mutation in the BRCA gene, being $15(65.2 \%)$ in BRCA 1, and 8 (34.8\%) in BRCA 2. All participants were female, with mean age of 46 years, and mean of three cases of cancer in the family. Seven (30.4\%) risk-reducing bilateral or contralateral mastectomy procedures were performed. The mean age at the performance of surgery was 38 years. There were six cases of previous breast carcinoma in one of the breasts, and one patient who had not been diagnosed with breast cancer. All of them were submitted to immediate reconstruction with inclusion of a silicone prosthesis. Laparoscopic bilateral salpingo-oophorectomy was performed in three patients. The histological type in the six patients with cancer was invasive carcinoma of no special type, being four triple negatives. Neoadjuvant chemotherapy was performed in four cases; in 6 cases, the mutation was in BRCA1. All patients remain without complains and free of disease, with mean follow-up of 29.5 months. Twelve (52.1\%) patients are being followed-up with mammography, resonance and annual clinical examination, and did not accept risk-reducing mastectomy or salpingo-oophorectomy. Conclusions: Risk-reducing mastectomy was performed in $30.4 \%$ of the patients with deleterious mutation of BRCA. The main mutation was BRCA, and in $85.7 \%$ of the cases, the patient presented with previous breast cancer in one of the breasts. 